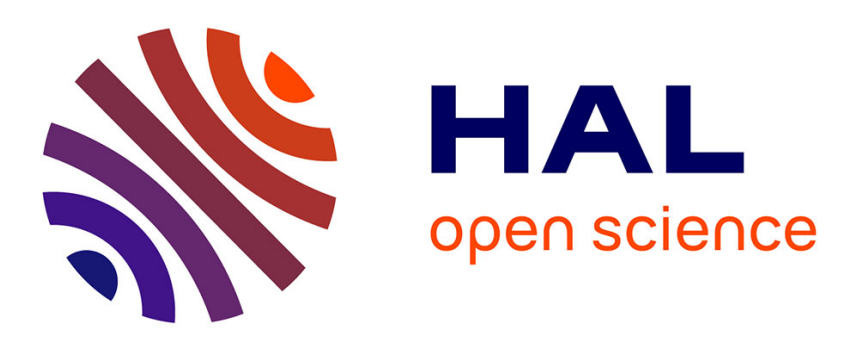

\title{
A Block-Based Edge Partitioning for Random Walks Algorithms over Large Social Graphs
}

\author{
Yifan Li, Camelia Constantin, Cedric Du Mouza
}

\section{To cite this version:}

Yifan Li, Camelia Constantin, Cedric Du Mouza. A Block-Based Edge Partitioning for Random Walks Algorithms over Large Social Graphs. 32ème Conférence sur la Gestion de Données - BDA2016, Nov 2016, Poitiers, France. hal-01398195

\section{HAL Id: hal-01398195 \\ https://hal.science/hal-01398195}

Submitted on 21 Nov 2016

HAL is a multi-disciplinary open access archive for the deposit and dissemination of scientific research documents, whether they are published or not. The documents may come from teaching and research institutions in France or abroad, or from public or private research centers.
L'archive ouverte pluridisciplinaire HAL, est destinée au dépôt et à la diffusion de documents scientifiques de niveau recherche, publiés ou non, émanant des établissements d'enseignement et de recherche français ou étrangers, des laboratoires publics ou privés. 


\section{A Block-Based Edge Partitioning for Random Walks Algorithms over Large Social Graphs}

\author{
Yifan LI \\ LIP6, University Paris 6 \\ Paris, France \\ yifan.li@lip6.fr
}

\author{
Camelia Constantin \\ LIP6, University Paris 6 \\ Paris, France \\ camelia.constantin@lip6.fr
}

\author{
Cedric du Mouza \\ CEDRIC Lab. - CNAM \\ Paris, France \\ dumouza@cnam.fr
}

\begin{abstract}
Recent results $[5,9,25]$ prove that edge partitioning approaches (also known as vertex-cut) outperform vertex partitioning (edge-cut) approaches for computations on large and skewed graphs like social networks. These vertex-cut approaches generally avoid unbalanced computation due to the power-law degree distribution problem. However, these methods, like evenly random assigning [25] or greedy assignment strategy [9], are generic and do not consider any computation pattern for specific graph algorithm. We propose in this paper a vertex-cut partitioning dedicated to random walks algorithms which takes advantage of graph topological properties. It relies on a blocks approach which captures local communities. Our split and merge algorithms allow to achieve load balancing of the workers and to maintain it dynamically. Our experiments illustrate the benefit of our partitioning since it significantly reduce the communication cost when performing random walks-based algorithms compared with existing approaches.
\end{abstract}

\section{General Terms}

Algorithms, Theory, Performance

\section{Keywords}

graph partitioning, social networks, random walk algorithms

\section{INTRODUCTION}

Random walks-based algorithms, such as personalized PageRank (PPR) [10] and personalized SALSA [4] have proven to be effective in personalized recommender systems due to their scalability. Some recent proposals rely on multiple random walks started from each vertex on graph, e.g. Fully personalized PageRanks computation using Monte

(c) 2016, Copyright is with the authors. Published in the Proceedings of the BDA 2016 Conference (15-18 November, 2016, Poitiers, France). Distribution of this paper is permitted under the terms of the Creative Commons license CC-by-nc-nd 4.0.

(c) 2016, Droits restant aux auteurs. Publié dans les actes de la conférence BDA 2016 (15 au 18 Novembre 2016, Poitiers, France). Redistribution de cet article autorisée selon les termes de la licence Creative Commons CCby-nc-nd 4.0.

BDA 2016, 15 au 18 Novembre, Poitiers, France.
Carlo approximation [3]. We call this intensive computation Fully Multiple Random Walks(FMRW).

Graph partitioning is a key area of distributed graph processing research, and plays an increasingly important role in both vertex-centric computation, like in Pregel model, and query evaluation. Recent results exhibit that edge partition(vertex-cut) turned out to be more efficient $[5,9]$ than traditional vertex partitioning(edge-cut) for computation on real-world graphs like social networks. As a consequence, several popular graph computation systems based on this approach have emerged, such as PowerGraph (GraphLab2) [9] and GraphX [25]. However their graph partitioning strategies are generic and do not depend on the algorithms performing the different computations. So they distribute edges evenly over partitions either randomly, i.e. a hash function of vertex ids in Giraph [2] and GraphX, or using a greedy or dynamic algorithm like in PowerGraph and GPS [19]. Besides, in contrast with light-weight algorithms like PageRank whose messages transmitted between vertices are only rank values, the simulation of heavy-communication algorithms, such as fully (multiple) random walks in this paper, have a more important communication cost since $(i)$ some extra path-related information of walks must also be delivered, and (ii) more than one message (walk) start from each vertex at one time. In this case, reducing communication cost is crucial for computation performance guarantee. particularly in a large scale computing cluster with complex machine-to-machine network infrastructure, or in distributed Computing-in-Memory system like Spark where communication might be a bottleneck for reaching high overall performance.

We propose in this paper a novel block-based, workloadaware graph(-edge) partitioning strategy which provides a balance edge distribution and reduce the communication costs for random walks-based computations. To the best of our knowledge, this is the first time a partitioning strategy dedicated to fully multiple random walks algorithms is proposed in Pregel model. Finally, the experiments show that our partitioning made significant improvements on both communication cost and time overhead.

\section{Contributions}

In summary, our contributions are:

1. a block-based partitioning strategy which considers graph algorithms specificities and the topological properties of real-world large graphs along with a seeds selection algorithm for building the blocks;

2. algorithms for merging and splitting blocks to achieve 
a dynamical load-balancing of the partitions;

3. an experimental comparison of our partitioning approach with several existing methods, over large real social graphes.

After the related work introduced in Section 2, Section 3 presents our block building strategy while Section 4 describes our blocks merge and refinement algorithms. Section 5 presents our experimental results and Section 6 concludes and introduces perspectives.

\section{RELATED WORK}

Pregel [15] has become a popular distributed graph processing framework due to the facilities it offers to the developers for large-graph computations, especially compared with other data-parallel computation systems, e.g. Hadoop. Pregel is inspired by Bulk Synchronous Parallel [23] computation model where computations on a graph consists of several iterations, also called super-steps. During a super-step, each vertex first receives all the messages which were addressed to it by other vertices in the previous super-step. Each vertex performs the actions defined by user-specific function, namely vertex.compute() [19] or vertex.program() [9], in parallel, using the updated values received in the messages. Then each vertex may decide to halt computing or to pass to other vertices the messages to be used in next super-step. When there is no message transmitted over graph during a super-step (i.e. every vertex has decided to halt) the computation stops. Due to Pregel success, several optimizations have been recently proposed in literature like the function Master.compute() [19] to incorporate global computations or Mirror Vertices [14] to reduce communication.

Traditional graph partitioning methods from 2-way cut by local search to multi-level approaches, like KernighanLin [12], PageRank Vectors [1] and METIS-based [11] algorithms, follow a vertex-partitioning (edge-cut) strategy. They propose partitionings which assign (almost) evenly vertices between partitions while minimizing the number of edges cut (edges between two partitions). These algorithms are efficient for small graphs, which are not sensitive to workload-unbalanced computation. However for real world graphs the large size and the power-law distribution lead to an unbalanced load over edge-cut partitions. More recent partitioning proposals in Pregel-like systems, such as Giraph, GPS, Gelly and Chaos[18] shard the graph using an edge-cut strategy which also generates unbalancing for power-law graphs, as introduced in [9].

While there exists a large literature and several implementations for vertex-partitioning, few recent works propose edge-partitioning. The two principal ones are GraphX [25] and PowerGraph [9]. However GraphX only offers random/hash partitioning where edges are evenly allocated over partitions with some constraints of communication between nodes. The underlying graph property, like local communities in social networks, is not properly explored. Unlike the hash-like partitioning, PowerGraph uses a heuristic partitioning method, Greedy Vertex-Cuts, which has shown significant better performance than random placement in any cases [9]. However, it also ignores the graph topological property and only focus on how to minimize the future communication on previous partitioning situation during edges distribution among partitions. Additionally, unlike our proposal, GraphX and PowerGraph partitionings can not be updated dynamically with graph evolution.

Our approach also takes advantage from the existence of communities. In [8] authors state that, due to the heavytailed degree distributions and large clustering coefficients properties in social networks, considering only the direct neighbors of a vertex allows to construct good clusters (communities) with low conductance. In [24] authors improve this method to detect communities over graph, but neither edge partitioning nor workload balancing problem is studied. Moreover, the overlapping communities approach for graph partitioning are not suitable to Pregel-like systems.

\section{BLOCK-BASED GRAPH PARTITION- ING}

\subsection{Principle}

Most existing edge partitioning methods, like random[25] or greedy [9] approaches achieve a balanced workload, which means each partition has the same number of edges. Our objective is to go beyond workload balancing and to lower graph processing time by reducing the communication between partitions during graph computation. In edgepartitioning approach, a vertex is possibly allocated to multiple partitions and communications between partitions occur when updating the different replicas (mirror vertices) at each Pregel super-step. Consequently, Vertex Replication Factor(VRF) firstly defined in [9] is often used as a communication measurement. So, given an edge partitioning, the communication cost is generally estimated in Pregel, as

$$
\text { cost }_{\text {Comm }}=O(L *(V R F *|V|))
$$

where $L$ is the number of supersteps (iterations) during graph computation.

However, in most real graphs, like social networks, there exist many clusters (communities). Our objective is to take advantage of this topological characteristic in our block construction. Local Access Pattern(LAP) is described in [26] for first time as one of three kinds of query workload in graphs. We propose to rely on its principles and analysis when proposing our edge-partitioning strategy for random walks-based algorithms considering graph communities to reduce communication costs.

As a consequence we consider that, while VRF is a good estimator of communication cost for some graph algorithms, it is not suitable for the random walks-based algorithms which follow a LAP, since the number of visits of each vertex is different for these algorithms in one super-step. In other words, communications are conducted unevenly on graph. So our objective is to design a new edge partitioning strategy dedicated to random walks-based algorithms which takes into consideration both the power-law topology of the graph and the LAP characterizing these algorithms. 


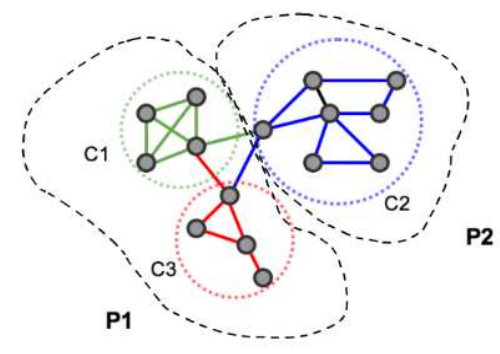

Figure 1: Example of blocks and partitions

In this way, we give a more exact expression for communication cost of algorithm algo during computing graph $G$ :

$$
\sum_{v \in V} N V(v) * \operatorname{Rep}(v)
$$

Where $N V(v)$ is the number of visits to vertex $v$ during algorithm simulation, and $\operatorname{Rep}(v)$ is the replication factor of $v$. Note that in this formulation, the size of message for communication is not considered. Hence, we could reduce the communication overhead by 1) cutting down the VRF in total as other existing approaches, and 2) making as few mirrors as possible(to obtain low $\operatorname{Rep}(v)$ ) for high- $N V(v)$ vertices in the simulation of specific graph algorithm, Random Walks here. The experimental results show that our block-based partitioning method can achieve both of them.

\section{Our approach}

A block corresponds to a tightly knit cluster in graph, e.g. a community in social network. In the Pregel approach, we consider the block as a set of edges which are "close" one to another, and these blocks become the component units of each partition in computation, but also the allocation units for workload over machines. Similar to the methodology adopted in vertex partitioning $[8,24]$, we propose to compute a set of $K$ blocks by exploring the graph. An edge is allocated to a block based on its distance from this block. We start a breadth-first search exploration (BFS) from a pre-defined set of $K$ seeds. For each edge encountered we update its distance with respect to all blocks. When the exploration step ended, we allocate the edges to the closest block.

Example 1. Figure 1 presents an example which illustrates our 2-step approach. First, we group edges based on their distances to the different seeds (here three seeds). We get three communities(edge blocks): c1, c2 and c3. Then we merge blocks to get partitions with similar size. Here we build partition P1, composed of $c 1$ and c3, and partition P2 corresponds to the single block c2.

\subsection{Distance of an edge}

In graph computation, how to measure the closeness between a pair of nodes is a fundamental question and it has been studied in many existing works. One interest of these distance measures is to detect cluster in graph (see Section 3.3). But based on the observation that for several graph algorithms like random walk, nearest neighbors, breadth-first search, etc, the communications during computations mainly occur between vertices belonging to the same cluster, several approaches extended this cluster detection to perform graph partitioning. For instance [1] proposed a PageRank vector method to find a "good" partition w.r.t. an initial vertex and several pre-set configurations. Besides, there are some proposals like [20] which describes how to obtain these partitions by conducting random walks.

For our edge-partitioning approach, we propose here to estimate the distance between an edge and a query vertex. We adapt the inverse P-distance[10] used for distance computation between two vertices.

\section{Vertex to vertex distance}

Inverse P-distance captures the connectivity: the more numerous and short paths between two vertices, the closer they are in graph topology.

So, the distance $\operatorname{dist}_{v}(i, j)$ from vertex $i$ to vertex $j$ in a directed graph $\mathrm{G}$ can be calculated by the paths between them, as follows:

$$
\operatorname{dist}_{v}(i, j)=\sum_{p \in P_{i j}} S(p)
$$

where the $P_{i j}$ denotes the set of paths from $i$ to $j . S(p)$ is the inverse distance value of path $p$ defines below.

According to the idea of inverse P-distance, we introduce the concept of "reachability" into distance computation between vertices. The reachability means the probability for a random walk starting from $i$ to arrive at $\mathrm{j}$. So, for path $p$ : $v_{0}, v_{1}, \ldots, v_{k}$ with length $k, S(p)$ can be defined by:

$$
S(p)=(1-\alpha)^{k} \cdot \prod_{i=0}^{k-1} \frac{1}{\text { outDeg }\left(v_{i}\right)}
$$

where $\alpha \in(0,1)$ is the teleporting probability, i.e., the probability to return to the original vertex, and outDeg $\left(v_{i}\right)$ is the out-degree of vertex $v_{i}$.

\section{Vertex to edge distance}

Based on the vertex to vertex distance introduced above, we define a vertex to edge distance. We adopt the following definition:

Definition 1 (EDGE Distance). The distance dist $_{e}(a, b)$ from a vertex a to an edge $b=(i, j)$ is:

$$
\operatorname{dist}_{e}(a, b)=\theta\left(\operatorname{dist}_{v}(a, i), \operatorname{dist}_{v}(a, j)\right)
$$

where $\theta$ is an aggregation function. 
In our experiment we choose the average function for $\theta$ but other functions like min or max may also be considered.

\subsection{Edges allocation algorithm}

Based on our edge distance we can now design an edge allocation algorithm. Our algorithm can be decomposed into three steps:

i) selection of a subset of vertices, namely seeds

ii) distance computation from each edge to all the seeds

iii) edges allocation to the different blocks partitions. However we argue that we can achieve a better partitioning when setting this number to a larger value. The reasons are:

- the expansion of each block can be processed independently, thus can be deployed easily on Pregel-like architecture;

- the combination of small blocks needs much less overhead cost than splitting (i.e., refinement) of large blocks when trying to minimize the replication factor;

- the more blocks we pre-computed, the higher the level of reusability of our partitioning will be.

\section{Distance computation}

\section{Seeds selection}

We consider for our block-partitioning a seed-expansion strategy: we select a vertex as seed for each block and add each edge to one of the existing block. Obviously the result of the partitioning, in term of size-balancing or communication during the computation, is highly dependent on the choice of the seeds. This problem has been studied in literature for instance in [24] to detect communities on graph or in [7] where authors propose and experiment for the precomputation step of their recommendation algorithm several landmark selection strategies.

Here we adopt the simple but efficient seeds selection procedure, based on Spread Hubs method (see [24]), which can be easily deployed on existing graph processing systems. There are two main measurements we used in seeds selection: 1) vertex degree, and 2) distance to other existing seeds. Our seeds selection algorithm is:

1. first we sort the vertices in descending order, according to their global (in+out) degrees;

2. then we scan the sorted list of vertices, and check if the current one is not too close to any existing seed, otherwise we discard it.

The rationale for this algorithm is that a vertex with a large global degree is a vertex with a centrality property and its connected vertices are likely to join its block. Moreover observing a minimum distance between seeds allows a better distribution of the seeds within the graph. Since BFS is efficiently implemented in Pregel, we use it to measure the distance between seeds. So we start a BFS from the seed candidate and report the number of hops required to reach the first existing seed. We observe experimentally that we achieve a good partitioning with this algorithm even when the depth of each seed's BFS is set 1 (so a new seed is not allowed to be the direct neighbor of an existing seed).

\section{Number of seeds.}

In our approach, each seed will determine a block which implies to have at least as many seeds as the number of final
For the second step of our algorithm, we compute first the inverse p-distance of each vertex to all seeds. To perform this distance computation efficiently in our Pregel-like architecture, we proceed to a parallel BFS exploration starting from each seed. Consider a set of seeds $\mathcal{S}=\left(s_{1}, s_{2}, \ldots, s_{N}\right)$. We maintain for each vertex $\nu$ a distance vector $\operatorname{dist}(\nu)=$ $\left(d_{1}, d_{2}, \ldots, d_{N}\right)$ where $d_{i}=\operatorname{dist}_{v}\left(s_{i}, \nu\right)$ is the inverse pdistance to the seed $s_{i}$. This vector is updated for each vertex encountered during the BFS exploration.

Since the BFS exploration in large graphs is very costly, we propose to limit the distance of exploration during the BFS. Indeed we observe in most of the large graphs (like social graphs) a community phenomenon which we capture by selecting the seeds among the vertices with the largest degrees, representing the center of these communities. Intuitively, the distance from the community center is short to other vertices inside the community. Actually, from our experiment results and "Six Degrees of Separation" theory [16], we observe that the radius of block, i.e., the distance from seed to potential community members is small and consequently the BFS depth can be set to a small value. social network, we found the vertex/edge coverages of 200 seeds can reach around 88 percent and 96 percent by limiting the BFS only to 3 and 4 hops respectively.

Finally we compute a distance vector for each edge in the graph. Consider an edge $e\left(\nu, \nu^{\prime}\right)$ and the distance vectors for its vertices $\operatorname{dist}(\nu)=\left(d_{1}, d_{2}, \ldots, d_{N}\right)$ and $\operatorname{dist}\left(\nu^{\prime}\right)=$ $\left(d_{1}^{\prime}, d_{2}^{\prime}, \ldots, d_{N}^{\prime}\right)$. Based on Definition 1 we compute the edge distance vector $\operatorname{dist}(\varepsilon)=\left(D_{1}, D_{2}, \ldots, D_{N}\right)$ as:

$$
\forall i \in[1 . . N], D_{i}=\operatorname{dist}_{e}\left(s_{i}, \varepsilon\right)
$$

\section{Edges allocation}

Finally we can allocate the different edges to the blocks according to their edge distance vector. We decide that an edge belongs to the block whose seed is the closest to this edge. For edges without any distance value (which means its end vertices have not been reached by any seed during the BFS step), we allocate them in an extra-block.
For instance, during the experiment on Livejournal [13] 


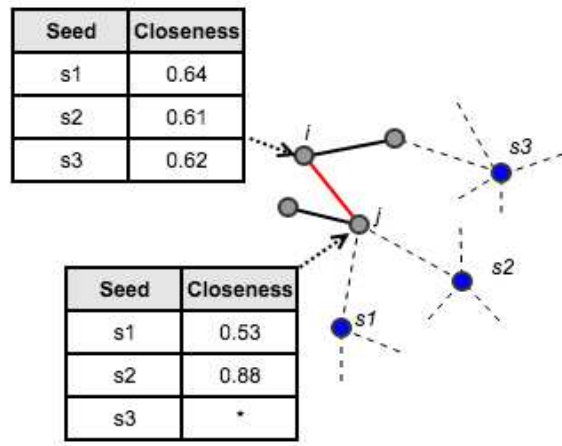

Figure 2: Example of edge allocation

EXAMPLE 2. We illustrate the edge allocation process with the example in Fig. 2.

We assume we have already computed the vertex distance vectors for vertices $i$ and $j$, considering three seeds s1, s2 and s3. Notice that the '*' value means that the current vertex can not be reached by the seed $s_{3}$ in our BFS exploration step. We sum (or make the average) the two vectors to determine the edge distance vector for $e(i, j): \operatorname{dist}(i, j)=$ $(0.64+0.53,0.61+0.88,0.62+0.0)=(1.17,1.49,0.62)$. Here we can clearly point out that the edge e should be allocated to s2 since it has maximum closeness value to this seed.

Observe that some optimizations are possible for storing the vertex distance vectors and for the edge distance vector computation. For instance we can avoid keeping all distance values to every seed, since in this edge allocation step, only the maximum value is used to allocate an edge to a block. So we could keep only a top- $k$ values for each vertex, with $k \leq|\mathcal{S}|$. Of course the larger $k$ is, the more precise our final result is.

\section{BLOCKS MERGE AND REFINEMENT ALGORITHMS}

Our block partitioning respects the topological properties of the (social) graph, e.g. local communities and power-law degree distribution to significantly reduce the communication costs compare to a random allocation strategy.

Given a number of servers $P$, we must determine how to allocate the different blocks to these servers considering two criteria:

- minimizing the global communication cost;

- balancing the storage and computation workload between servers.

These conditions can be captured by the following definition.

DEFINITION 2 (BALANCED EDGE PARTITIONING). Consider a graph $G(V, E)$ where $V$ is the set of vertices and $E$ the set of edges, a set of blocks $\mathcal{B}$ and a number of servers
P. The balanced edge partitioning $\mathcal{A}(\mathcal{B}, P)$ is defined as:

$\mathcal{A}(\mathcal{B}, P) \in 2^{\mathcal{B}}$, such that $\left\{\begin{array}{l}\forall \mathcal{A}^{\prime} \in 2^{\mathcal{B}}, \frac{1}{|V|} \sum_{v \in V}|\operatorname{alloc}(v, \mathcal{A})| \\ \leq \frac{1}{|V|} \sum_{v \in V}\left|\operatorname{alloc}\left(v, \mathcal{A}^{\prime}\right)\right| \\ \forall i \in[1 . . P], \eta \frac{|E|}{P} \leq\left|\operatorname{Edge}\left(p_{i}\right)\right| \\ \leq \lambda \frac{|E|}{P}\end{array}\right.$

where $p_{i}$ is a partition (server) and Edge $\left(p_{i}\right)$ the edges it contains, alloc $(e, \mathcal{A})$ is the set of partitions to which edge e is assigned with the partitioning $\mathcal{A}$ (more than one if the vertex is replicated) and $(0 \leq \eta \leq 1 \leq \lambda)$ are small constants to control the storage in each partition.

The first part of the definition means the partitioning $\mathcal{A}$ is the one which minimizes the Vertex Replication Fac$\operatorname{tor}(\mathrm{VRF})$. The VRF measure adopted for instance in [9] means the less partitions the vertex span on average, the less communication across partitions the system initiates for vertices synchronization before running into the next superstep. The second part of the definition allows to control the size of a partition to fit the server capacity and to have an almost balanced edges distribution.

With respect to Definition 2 we can proceed to the final partitioning based on the different blocks we built.

\section{Block split}

Since the edges allocation to blocks is only based on a distance criterium some blocks may not fit the maximum size allowed for a partition (second part of Definition 2). Consequently we propose a simple split strategy. Assume that the size of a partition $p_{i}$ is $(\beta-1) \lambda \frac{|E|}{P} \leq\left|E d g e\left(p_{i}\right)\right|<\beta \lambda \frac{|E|}{P}$. We then apply our block building algorithm to the partition $p_{i}$ with $\beta$ seeds to split it into $\beta$ sub-blocks. We potentially iterate the process for any of the sub-blocks which exceeds the partition size.

\section{Blocks merge}

Our block building may also result in producing some blocks whose size is lower than the minimal size (i.e. $\eta \frac{|E|}{P}$, see Definition 2). For such a block we re-allocate its edges without considering its seed anymore. Observe that this may lead in turn to some block splits.

\section{Block allocation}

We assume that, possibly after some required splits, the size of all blocks respect the partition size limit. To allocate the blocks to the different partitions, two strategies may be considered: based only on the balancing of the partition sizes, or on minimizing the replication factor between partition. 
Considering this latter approach, we exhibit the following drawbacks: (1) there is an exponential complexity for finding the best blocks allocation considering this criterium, (2) the final size of each partition may highly differ one from another, (3) reducing the global replication factor will not reduce that much the cost of the random-walks algorithms since a path starting in one block and finishing in another is unlikely (according to our blocks building) and finally (4) this partitioning could not evolve dynamically and the partitioning must be re-built when many edges are added or removed.

Consequently we decide to adopt a blocks allocation considering only the size criterium, to achieved a balanced partitioning. We propose a simple but efficient greedy algorithm. We allocate the largest block to the partition with the smallest size, and we iterate this strategy until all blocks are allocated. Consequently this allocation is in $O(|\mathcal{B}|)$ where $\mathcal{B}$ represents the set of blocks.

The whole algorithm is presented in Algorithm 1 where split refers to a function which proceeds to the block split introduced above, sortSize is a function which sorts a set of blocks according to their size, from the largest to the smallest one, and first returns the first element from an ordered set.

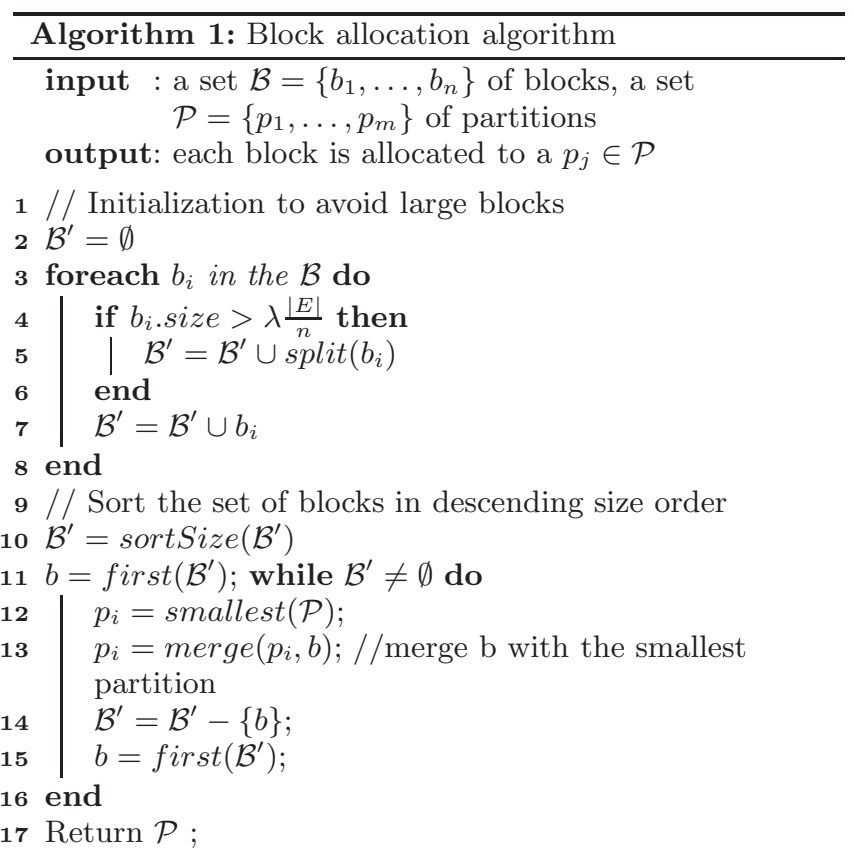

\section{Managing graph dynamicity}

Large graphs, especially for social network applications, are often characterized by a high dynamicity. One important aspect of our partitioning algorithm is its ability to manage this dynamicity. Indeed when adding a new edge (for instance when adding a friend on Facebook or an url on a Website) we simply have to aggregate the two vertex distance vectors of the two vertices of the edge if both ver- tices were already present in the graph to compute its edge distance vector. Then we allocate the edge to the block, and consequently to the partition, with the highest distance score. If one of the vertex is new, we have first to perform the BFS exploration from that vertex and compute its vertex distance vector. Potentially this edge allocation may lead to a block split which can be handled with our split algorithm. Oppositely when removing an edge, the size of a block may become too small and we proceed to our block merge algorithm.

\section{EXPERIMENTS}

This section presents experiments on our block-based partitioning strategy. We compare it with existing edge partitioning methods: the hash-based approaches [25] and greedy algorithm [9].

\subsection{Setting}

Computation are performed using GraphX [25] APIs in Spark [27] (version 1.3.1), on a 16 nodes cluster. Each machine has 22 cores with 60 GB RAM running Linux OS. For our experiments we set teleporting probability $\alpha$ to a classical value 0.15 . The depth of the BFS exploration (i.e., the maximum length considered for paths from seed to other vertices) is set to 4 . So we intend to compute only the nearby vertices to current seed, rather than to every vertices in graph, according to Six Degrees of Separation theory[17].

Data Sets. We validate our approach on two datasets: LiveJournal [6] with $4.8 \mathrm{M}$ vertices and $68.9 \mathrm{M}$ edges, and Pokec [22] with $1.6 \mathrm{M}$ vertices and $30.6 \mathrm{M}$ edges. These datasets can be downloaded from $S N A P^{1}$.

\section{Competitors}

Hash Partitioning. There are four wide used random(hash)like partitioning methods ${ }^{2}$, introduced in GraphX:

- RandomVertexCut: allocates edges to partitions by hashing the source and destination vertex IDs.

- CanonicalRandomVertexCut: allocates edges to partitions by hashing the source and destination vertex IDs in a canonical direction.

- EdgePartition1D: allocates edges to partitions using only the source vertex ID, co-locating edges with the same source.

- EdgePartition2D: allocates edges to partitions using a 2D partitioning of the sparse edge adjacency matrix.

Greedy Vertex-Cuts. PowerGraph proposes a greedy heuristic for edge placement process which relies on the previous allocation of vertices to determine the partition next edge should be assigned.

\footnotetext{
${ }^{1}$ https://snap.stanford.edu/data/index.html

${ }^{2}$ see details and implementations at http://spark.apache.org/docs/latest/api/scala/index.html
} 


\section{Graph Algorithms.}

Except Fully Multiple Random Walks(FMRW), the highcommunication graph algorithm we mentioned before, some other algorithms are also conducted to estimate computation performance, i.e., runtimes, with our partitioning:

- Fully Multiple Random Walks(FMRW): in some complicated graph applications, such as ranking and recommendation (like the fully Personalized PageRank(PPR) computation in $[3,21])$, simulating multiple random walks from each vertex is the staple and a CPU/IO intensive task. For instance, in the following experiments, we launch two independent random walks of length 4 starting from each vertex. For longer walks, we can obtain them by combining short ones like in $[3,21]$. Thus we have 4 supersteps(iterations) in total graph computation, and in each superstep, every walk at that vertex will be randomly delivered to one of its direct neighbours, here note that the restart operation is not introduced in. Finally we can gain $|V| * 2$ random walks after all supersteps are finished.

- PageRank: the most used algorithm to evaluate graph processing system, in which the messages transmitted between vertices are pure digital values. It means the $\mathrm{I} / \mathrm{O}$ overhead is not very high since the size of message is small even the number of messages is big. Particularly in our experiments, we conduct both static and dynamic versions. The number of supersteps is fixed in former, to control the overall time-consuming of graph computation. Instead of it, a convergence tolerance, e.g. 0.005 or 0.001 , is applied to the latter, which is better to used for required computation result accuracy.

- Connected Components: an algorithm to compute the connected component for each vertex that can be used to approximate the cluster structures in graph, in other words, we can say it is a locality-sensitive algorithm. The procedure of its computation is simple, in which each vertex keeps a component id, e.g. the lowest id received from neighbours, then in each superstep it would update the id according the latest received messages.

\subsection{Communication}

Our approach aims at reducing the runtime graph processing thanks to a significant reduction of the communication costs.

\subsubsection{Vertex Replica Factor (VRF)}

VRF is the traditional way to compare two partitionings regarding the communication costs, independently of the algorithm executed. We compare the VRF of our Block-based partitioning with the one of the competitors for different numbers of partitions. Results are depicted on Figure 3. We observe that, as observed in [9], partitioning strategies
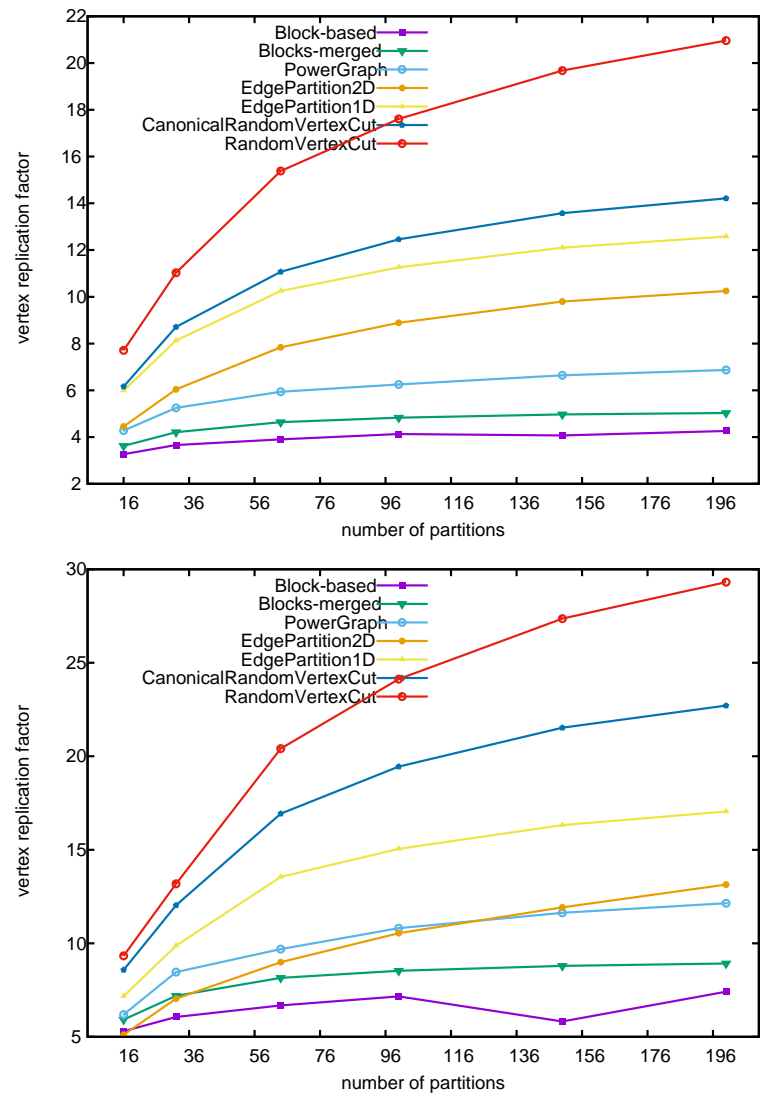

Figure 3: VRF w.r.t. edge partitioning methods on LiveJournal (above) and Pokec (below) 
based on topology outperform as expected hash-based methods: VRF decreased by 30-60\% (resp. 60-80\%) for Powergraph (resp. our block strategy) compare to the strategies used in GraphX. This experiment also illustrates the benefit of our global approach for edge allocation compare to a greedy approach with on average a $40 \%$-lower VRF.

\subsubsection{Number of Messages}

VRF is a general criterium to compare two partitioning strategy independently from the algorithms, but we expect our partitioning to exhibit even better results for random walks-based algorithms. Consequently to estimate the benefit of our approach we simulate fully multiple random walks (FMRW) and we measure the number of messages exchanged between partitions. From each vertex we perform 2 random walks of length 4 and we report experimental results in Table 1. We observe that our method reduces significantly the number of messages exchanged between partitions. For instance with 100 partitions, 61.8 million messages are necessary for processing the FMRW with our method while 381.9 million are transmitted with Random-Vertex-Cut method, so a drop of $84 \%$. This result was expected since the VRF is 3-4 times lower with our method than with Random-VertexCut. But we notice that if the reduction of the number of messages and of the VRF were proportional, the system should exchange 89.4 million message. This $30 \%$ gain in the number of messages transmitted validates our intuition that random walks intend to stay in the local cluster(community). So low-replicated vertices (close to the seed in block) are accessed more times, and oppositely few random walks reach the farthest, high-replicated, vertices. Similar results are obtained from experiments on Pokec.

\subsection{Runtimes}

We propose to evaluate how the runtime of different graph processing algorithms benefits our partitioning, compared to other methods. First, we launch FMRW, a heavy-communication algorithm, on LiveJournal and Pokec datasets respectively, with 3 random walks of length 4 started from each vertex. From the results in Figure 4, we see that our partitioning can save up between from 20 to 65 percent of runtime, compared with other partitionings, for both datasets.

We also test our method with traditional PageRank algorithm. We consider the static (fixed number of iterations) and dynamical (with convergence and a threshold value) approaches. We consider there are 200 partitions and we proceed to resp. 30, 50 and 100 iterations for static PageRank and to dynamical PageRank with resp. 0.005 and 0.001 convergence factor. Figure 5 depicts results and confirms that our partitioning method outperforms other ones. While we observe a small 5-20\% gain for the static implementation of PageRank, we reach a 20-55\% gain for the dynamical implementation.

One notable thing here is the result of PowerGraph partitioning method on LiveJournal graph is worse than anticipated in static PageRank experiments, w.r.t its VRF score. One speculation is some of its partitions consume too much time in one or more supersteps(iterations of graph compu-

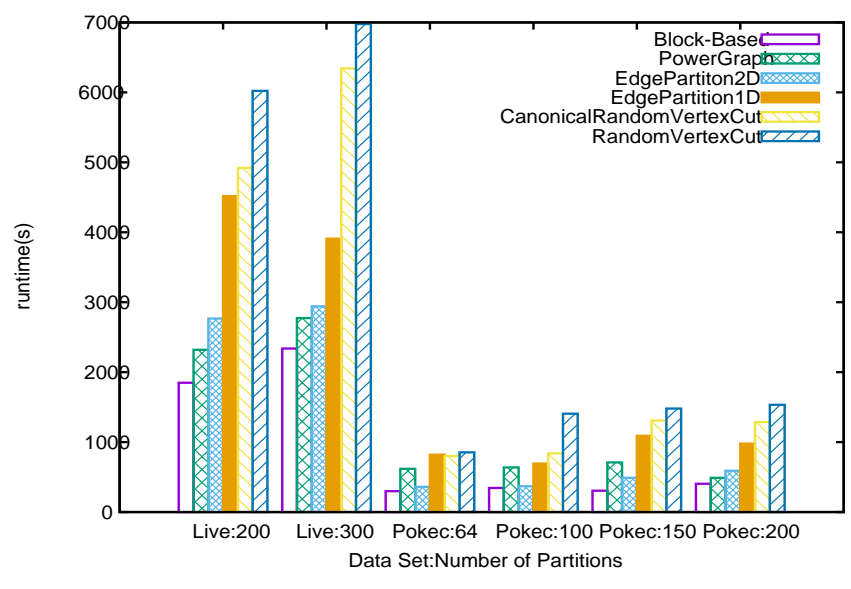

Figure 4: Runtimes for FMRW with different partitionings for LiverJournal and Pokec

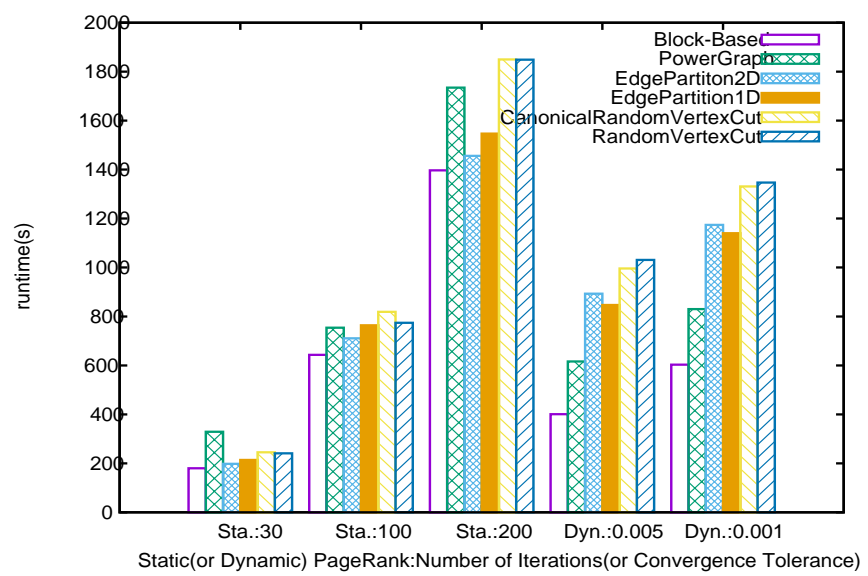

Figure 5: Runtimes for static and dynamic PageRank for LiverJournal

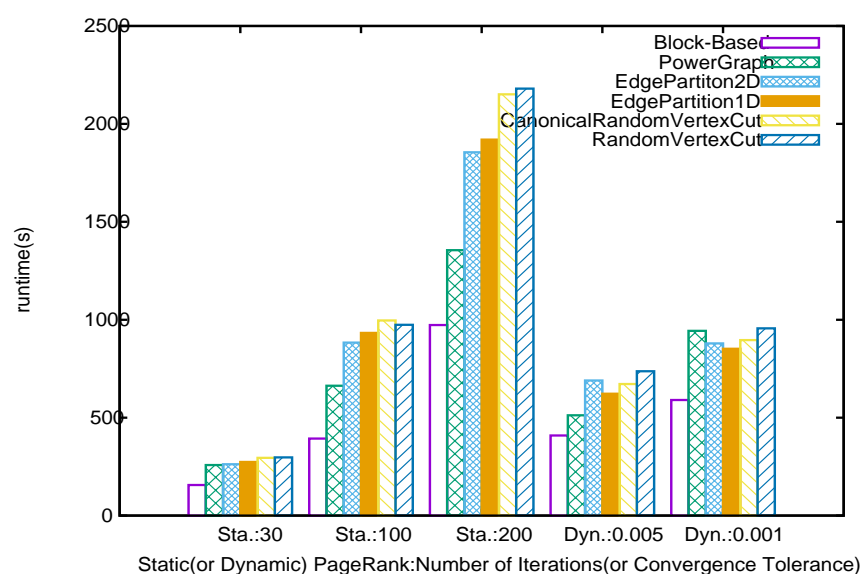

Figure 6: Runtimes for static and dynamic PageRank for Pokec 
Table 1: Messages transmitted in FMRW (LiveJournal)

\begin{tabular}{|r||r|r||r|r|r|r||}
\hline \multicolumn{1}{|c||}{} & \multicolumn{2}{c||}{ Random-Vertex-Cut[25] } & \multicolumn{4}{c||}{ Block-based partitioning } \\
\hline \#Partitions & VRF & real mess. & VRF & real mess. & expected mess. & ratio \\
\hline 64 & 15.38 & $303.5 \mathrm{~m}$ & 3.90 & $55.3 \mathrm{~m}$ & $76.9 \mathrm{~m}$ & 0.72 \\
\hline 100 & 17.61 & $381.9 \mathrm{~m}$ & 4.13 & $61.8 \mathrm{~m}$ & $89.4 \mathrm{~m}$ & 0.69 \\
\hline 150 & 19.68 & $464.8 \mathrm{~m}$ & 4.07 & $70.6 \mathrm{~m}$ & $96.1 \mathrm{~m}$ & 0.73 \\
\hline 200 & 21.12 & $525.6 \mathrm{~m}$ & 4.26 & $76.0 \mathrm{~m}$ & $106.0 \mathrm{~m}$ & 0.72 \\
\hline
\end{tabular}

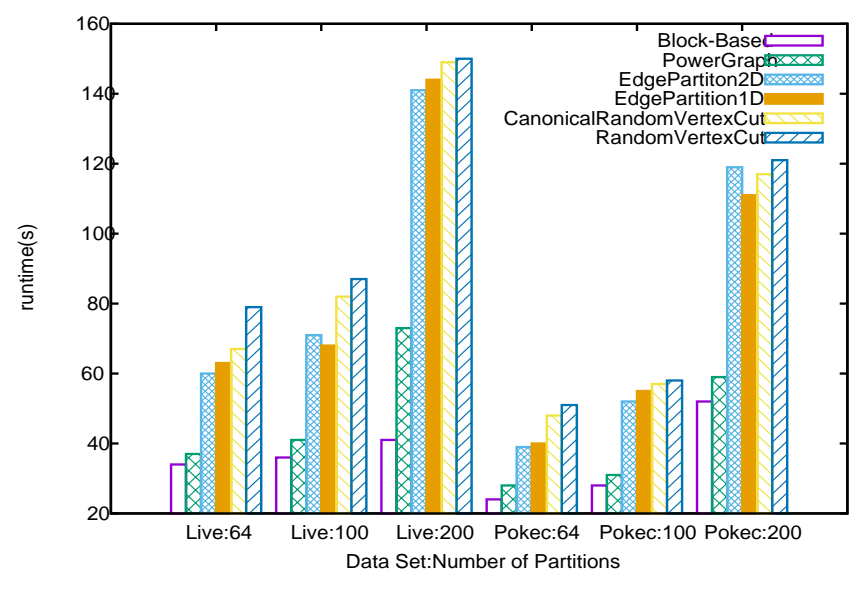

Figure 7: Runtimes for Connected Components(CCs) computation with different partitionings for LiverJournal and Pokec

tation) which leads to long runtime in total because other partitions need to wait them to finish that superstep. From the experiments on Pokec graph, we can see, in Figure 6, it works well for static PageRank.

In addition, we propose to use a locality-sensitive algorithm, Connected Components, to verify our partitioning works as expected. Finally, the results shown in Fig.7 have proven again that our partitioning method performs much better than others.

\section{CONCLUSION AND FUTURE WORK}

We present in this article a vertex-cut partitioning for random-walks-based algorithms relying on the topology to build blocks which respect local communities. We propose split and merge algorithms to get and to maintain the final partitioning. We experimentally demonstrate that our proposal outperforms existing solutions.

As future work we plan to investigate different seeds selection algorithms. While this problem has been studied in different context (see [24, 7]) we believe that the nature of the graph algorithms, here random walks-based algorithms, must be considered when selecting the seeds. We also intend to study the $5-10 \%$ of vertices which are not reached by the BFS exploration issued at seeds. They are located on the periphery of social graph and are poorly connected. While we currently place them to an extra-block, we will design a strategy to allocate them to existing blocks.

\section{REFERENCES}

[1] R. Andersen, F. R. K. Chung, and K. J. Lang. Local Graph Partitioning using PageRank Vectors. In Proc. of the IEEE Symposium on Foundations of Computer Science (FOCS), pages 475-486, 2006.

[2] Apache. Giraph.

[3] B. Bahmani, K. Chakrabarti, and D. Xin. Fast Personalized PageRank on MapReduce. In Proc. of the $A C M$ SIGMOD International Conference on Management of Data (SIGMOD), pages 973-984, 2011.

[4] B. Bahmani, A. Chowdhury, and A. Goel. Fast Incremental and Personalized PageRank. Proc. of the VLDB Endowment (PVLDB), 4(3):173-184, 2010.

[5] F. Bourse, M. Lelarge, and M. Vojnovic. Balanced Graph Edge Partition. In Proc. of the ACM SIGKDD International Conference on Knowledge Discovery and Data Mining (KDD), pages 1456-1465, 2014.

[6] F. Chierichetti, R. Kumar, S. Lattanzi, M. Mitzenmacher, A. Panconesi, and P. Raghavan. On Compressing Social Networks. In Proc. of the ACM SIGKDD International Conference on Knowledge Discovery and Data Mining (KDD), pages 219-228, 2009.

[7] R. Dahimene, C. Constantin, and C. du Mouza. RecLand: A Recommender System for Social Networks. In Proc. of the ACM International Conference on Conference on Information and Knowledge Management (CIKM), pages 2063-2065, 2014.

[8] D. F. Gleich and C. Seshadhri. Vertex Neighborhoods, Low Conductance Cuts, and Good Seeds for Local Community Methods. In Proc. of the ACM SIGKDD International Conference on Knowledge Discovery and Data Mining (KDD), pages 597-605, 2012.

[9] J. E. Gonzalez, Y. Low, H. Gu, D. Bickson, and C. Guestrin. PowerGraph: Distributed Graph-Parallel Computation on Natural Graphs. In Proc. of the USENIX Symposium on Operating Systems Design and Implementation (OSDI), pages 17-30, 2012.

[10] G. Jeh and J. Widom. Scaling Personalized Web Search. In Proc. of the International World Wide Web Conference ( $W W W$ ), pages 271-279, 2003.

[11] G. Karypis and V. Kumar. A Fast and High Quality Multilevel Scheme for Partitioning Irregular Graphs. SIAM J. Scientific Computing, 20(1):359-392, 1998.

[12] B. W. Kernighan and S. Lin. An Efficient Heuristic Procedure for Partitioning Graphs. The Bell System Technical Journal, 49(2):291-307, 1970.

[13] J. Leskovec, K. J. Lang, A. Dasgupta, and M. W. Mahoney. Community Structure in Large Networks: Natural Cluster Sizes and the Absence of Large 
Well-Defined Clusters. CoRR, abs/0810.1355, 2008.

[14] Y. Low, J. Gonzalez, A. Kyrola, D. Bickson, C. Guestrin, and J. M. Hellerstein. Distributed GraphLab: A Framework for Machine Learning in the Cloud. Proc. of the VLDB Endowment (PVLDB), 5(8):716-727, 2012.

[15] G. Malewicz, M. H. Austern, A. J. C. Bik, J. C. Dehnert, I. Horn, N. Leiser, and G. Czajkowski. Pregel: a System for Large-scale Graph Processing. In Proc. of the ACM Symposium on Principles of Distributed Computing (PODC), page 6, 2009.

[16] M. Newman, A.-L. Barabasi, and D. J. Watts. The Structure and Dynamics of Networks: (Princeton Studies in Complexity). Princeton University Press, 2006.

[17] M. Newman, A.-L. Barabasi, and D. J. Watts. The Structure and Dynamics of Networks: (Princeton Studies in Complexity). Princeton University Press, 2006.

[18] A. Roy, L. Bindschaedler, J. Malicevic, and W. Zwaenepoel. Chaos: Scale-out Graph Processing from Secondary Storage. In Proc. of the Symposium on Operating Systems Principles (SOSP), pages 410-424, 2015.

[19] S. Salihoglu and J. Widom. GPS: a Graph Processing System. In Proc. of the Conference on Scientific and Statistical Database Management (SSDBM), pages 22:1-22:12, 2013.

[20] P. Sarkar and A. W. Moore. Fast Nearest-neighbor Search in Disk-resident Graphs. In Proc. of the ACM SIGKDD International Conference on Knowledge Discovery and Data Mining (KDD), pages 513-522, 2010.

[21] A. D. Sarma, S. Gollapudi, and R. Panigrahy. Estimating PageRank on Graph Streams. In Proc. of the ACM SIGMOD-SIGACT-SIGART Symposium on Principles of Database Systems (PODS), pages 69-78, 2008.

[22] L. Takac and M. Zabovsky. Data Analysis in Public Social Networks. Present Day Trends of Innovations, pages 1-6, 2012.

[23] L. G. Valiant. A Bridging Model for Multi-core Computing. In Proc. of the Annual European Symposium Algorithms - ESA, pages 13-28, 2008.

[24] J. J. Whang, D. F. Gleich, and I. S. Dhillon. Overlapping Community detection Using Seed set Expansion. In Proc. of the ACM International Conference on Information and Knowledge Management (CIKM), pages 2099-2108, 2013.

[25] R. S. Xin, J. E. Gonzalez, M. J. Franklin, and I. Stoica. GraphX: a Resilient Distributed Graph System on Spark. In Proc. of the International SIGMOD Workshop on Graph Data Management Experiences and Systems (GRADES), page 2, 2013.

[26] S. Yang, X. Yan, B. Zong, and A. Khan. Towards Effective Partition Management for Large Graphs. In Proc. of the ACM SIGMOD International Conference on Management of Data (SIGMOD), pages 517-528, 2012.

[27] M. Zaharia, M. Chowdhury, T. Das, A. Dave, J. Ma, M. McCauly, M. J. Franklin, S. Shenker, and I. Stoica. Resilient Distributed Datasets: A Fault-Tolerant
Abstraction for In-Memory Cluster Computing. In Proc. of the USENIX Symposium on Networked Systems Design and Implementation (NSDI), pages 15-28, 2012. 